\title{
From biomedical big data to knowledge and action
}

\author{
Mei Chen ${ }^{1}$
}

Received: 10 October 2018 / Accepted: 17 October 2018 / Published online: 26 October 2018

(c) Springer-Verlag GmbH Germany, part of Springer Nature 2018

It is a great honor to serve as the lead guest editor for this special issue of Machine Vision Applications, Learning and Understanding of Biomedical Big Data. Advances in imaging technologies have enabled researchers and practitioners to acquire large volumes of biomedical images. This has made it possible to conduct large-scale, image-based experiments for biomedical discovery. The main challenge and bottleneck in such experiments are the conversion from "biomedical big data" to interpretable information and hence discoveries. Computer vision has huge potential for automating the analysis and understanding of such data. Not only do computers have more "stamina" than human annotators for such tasks, but they also perform analysis that is more reproducible and less subjective. Recent years, novel machine learning techniques, especially deep learning, have revolutionized many areas in computer vision and significantly advanced the stateof-art. This special issue serves to attract active researchers around the world to share their recent innovation on this exciting topic.

Those of us working in the field of biomedical image analysis have been well aware of the rapid growth in data and the important role of advances in computer vision and machine learning, but the papers in this issue provide greater insight into the diversity of the problems as well as the innovative approaches to tackle them. I learned a great deal from reading these papers and doing so increased my respect for and understanding of the larger challenge of working with biomedical big data.

As documented in this issue, the broad scope of contributions is truly impressive. The topics discussed herein range from segmentation, classification, tracking, event detection, image modality transfer to application and workflow. An efficient and robust hybrid method for segmentation of zebrafish objects from bright-field microscope images combined different methods of segmentation of zebrafish larval images into a hybrid procedure to improve both efficiency and accuracy; Improved deep learning based macromolecules

Mei Chen

may4mc@gmail.com

1 Microsoft, Redmond, WA, USA structure classification from electron cryo tomogram proposed three improved architectures of convolutional neural networks for the problem of classification of macromolecules in cryo-electron tomograms; A multi-seed dynamic local graph matching model for tracking of densely packed cells across unregistered microscopy image sequence demonstrated significant improvement over the existing local graph matching algorithm; Mitosis Event Recognition and Detection based on Evolution of Feature in Time Domain utilized the evolution of features in the time domain to represent the feature of mitosis events; Learning to Transfer Microscopy Image Modalities built joint normal distributions on the different types of images and then computed the conditional distributions to transfer the source images into the target ones; Single Sensor Hand-Vein Multimodal Biometric Recognition using Multiscale Deep Pyramidal Approach presented a fusion-based palmprint and fingerprint vein-based biometric system; Validation of Right Coronary Artery Lumen Area from Cardiac Computed Tomography against Intravascular Ultrasound developed a workflow for quantifying lumen area and coronary stenosis from CT images. The breadth of the problems being addressed is remarkable.

We employed the journal's standard procedures for accepting manuscripts for this issue. All submissions were subjected to the normal MVA peer-review process. Based on the peer reviews, we made the final decisions about acceptance or rejection. Editors Weidong Cai, Dimitris Metaxas, Anan Liu, Tolga Tasdizen, and Shaoting Zhang worked hard in organizing the special issue. We would like to express our gratitude to the many reviewers who provided invaluable feedback on the manuscripts, often through multiple iterations, and contributed to their quality. We would also like to thank the Editor-in-Chief, Rahul Sukthankar, for the guidance he provided during the editorial process of this special issue. We hope that readers will find these papers interesting, useful, and inspiring. As biomedical big data grow in quantity and diversity, we feel that their analysis and understanding can only grow in importance.

Happy reading. 
Mei Chen is a Principal Scientist at Microsoft's AI, Perception, and Mixed Reality group. From 2014 to 2018, she was an Associate Professor at the State University of New York, Albany. From 2011 to 2014, she built and led the Intel Science and Technology Center on Embedded Computing at Carnegie Mellon University, driving collaborations across four research themes involving seven universities including UC Berkeley, Cornell, UIUC, UPenn, Georgia Tech, UW, and Penn State. Previously she held researcher and research lead posi- tions at Intel Labs, Hewlett Packard Labs, and SRI Sarnoff Corporation. Mei's work in computer vision and biomedical image analysis were nominated finalists for six Best Paper Awards and won three. While at HP Labs, she successfully transferred her research in computational photography to five hardware and software products that went to market. She earned a Ph.D. in Robotics from the School of Computer Science, Carnegie Mellon University, and a M.S. and B.S. from Tsinghua University, Beijing, China. 